\section{ITINERARIOS CULTURALES Y PATRIMONIO MUNDIAL} PORRES, LIMA-PERÚ, 2010.

RESEÑA DE JOSÉ HAYAKAWA CASAS

\section{FONDO EDITORIAL DE LA UNIVERSIDAD DE SAN MARTÍN DE}

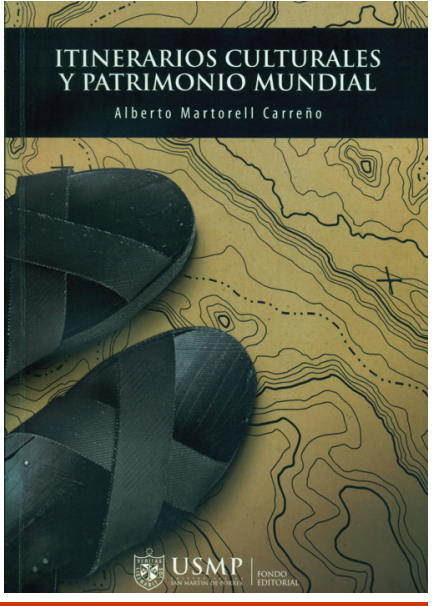

El libro Itinerarios Culturales y Patrimonio Mundial de Alberto Martorell Carreño se basa en su tesis doctoral (UNED, 2008), por la cual obtuvo la calificación Summa Cum Laude. La tesis parte de un vasto y profundo análisis de la evolución del concepto de patrimonio cultural y las categorías que se le han adicionado en el marco de la Convención del Patrimonio Mundial.

El tema abordado en la investigación referida es muy novedoso y bastante 'reciente' en el medio académico peruano. Con mayor precisión, identificamos como génesis la presentación del Camino de Santiago de Compostela (España), en 1993, y luego una reunión en 1994, aunque entonces aún no se manejaba con precisión los conceptos, ni las diferencias y especificidades entre itinerarios culturales e itinerario patrimonial, planteadas por parte de instituciones de referencia internacional tales como ICOMOS y UNESCO. Justamente un mérito evidente de este libro es que nos comparte la principal tesis del autor sobre esta categoría patrimonial, cuya nominación es la que defiende el libro: entender los itinerarios culturales a través de la categoría patrimonio, de la conectividad entre puntos geográficos distintos y la interrelación cultural.

Se trata de un libro que desarrolla un detallado análisis de la evolución histórica del concepto de patrimonio cultural, y sus categorías y acepciones más relevantes, en el marco de la Convención sobre la Protección del Patrimonio Mundial (CPPM). Asimismo, profundiza en el estudio científico y detallado de los itinerarios culturales como encuadre teórico-conceptual -pero también gestionario- y metodología para la identificación de este tipo de bienes, para luego analizar dos casos fundamentales para este nuevo horizon- te patrimonial: el Camino de Santiago de Compostela en España -también conocido como Camino Francés- en su especial condición de primer itinerario cultural inscrito en la lista de bienes del patrimonio mundial; y, el Qhapaq Ñan -también conocido como Camino Principal Andinoen su condición singular de ser el mayor proyecto promovido por los países andinos -con la asesoría técnica del Centro del Patrimonio Mundial de la Unesco- y uno de los más complejos del mundo por sus monumentales desafíos gestionarios.

La primera parte inicia revisando la evolución jurídica de los conceptos y de su normativa, así como la Convención sobre la Protección del Patrimonio Mundial. Analiza el concepto patrimonial a lo largo del tiempo -y especialmente en el marco de la Convención referida-, de los valores patrimoniales, sus escalas, los lineamientos para la inscripción de sitios y su respectiva gestión. De especial importancia es el acento en la apertura conceptual, que pasa del sitio puntual a una mirada más amplia, no solo geográfica, sino que incluye hechos y procesos históricos de intercambio cultural e interrelaciones humanas construidas a través del tiempo.

La segunda parte está centrada en el análisis, reflexiones y propuestas metodológicas en torno a los "bienes asociados a la movilidad de las personas, que proporcionan conectividad e interrelación" (p. 18) en su virtud de propiedades que permiten argumentar el reconocimiento de dichos espacios como itinerarios culturales.

Uno de los aportes más interesantes del libro está en la tercera parte, donde la atención se concentra específicamente en desarrollar el análisis de los dos ejemplos seleccionados: el Camino de Santiago -el cual fue su objeto de investigación doctoral- y nuestro entrañable Qhapaq Ñan. Ambos análisis cuentan con enorme detalle; aportan valiosa información acerca de sus características, naturaleza, valores, pasos para la nominación, entre otros aspectos. Se revisa críticamente los criterios utilizados en la normatividad aplicada a su gestión y a su protección. El caso del Camino de Santiago -primer itinerario inscrito como patrimonial mundial- ha sido indudablemente una excelente referencia para el esfuerzo desplegado en el Perú y otros países andinos para madurar la nominación internacional del Qhapaq Ñan. Justamente sobre este segundo caso, el libro sistematiza y analiza los distintos estudios y acciones en torno al mismo, y a este proceso que el Perú -bajo el liderazgo del Ministerio de Cultura- emprendió exitosamente, pues logró dicho reconocimiento mundial. Como la vocación del libro es analítico-crítica, se reflexiona acerca de los vacíos existentes en el expediente para la inscripción del Camino de Santiago, ya sea en la identificación del trazado y en las incompatibilidades informativas, como en la solución de una gestión unitaria merced a su categoría de patrimonio supranacional (normatividad y autoridad de nivel supranacional, para velar por todo el conjunto). La idea fue identificar 'lecciones aprendidas' aplicables no solo en el Qhapaq Ñan sino en todos los casos que decidan afrontar el mismo desafío gestionario patrimonial.

Finalmente, cabe destacar el carácter poliédrico de esta innovadora publicación, en el sentido de que aborda una categoría de patrimonio de las más complejas, no solo por su naturaleza, extensión, variedad, sino porque -como bien dice el autor- además del propio bien se valora la sinergia histórica gestada en los territorios y pueblos enlazados en torno al camino. Es decir, se valora además del propio bien a las relaciones desarrolladas en él y las expresiones culturales construidas en su uso social. Se suma la perspectiva jurídica patrimonial, no solo por la mirada disciplinar del autor sino por su especialización en el 'Derecho de la cultura', también de desarrollo reciente y novedoso entre nosotros.

Merced al minucioso trabajo científico de Alberto Martorell, esta publicación ya es un manual de consulta obligada en lo concerniente a los itinerarios culturales y representa un importante referente para los profesionales, estudiantes e instituciones vinculadas a lo cultural, el paisaje, el territorio y la gestión patrimonial, además del público interesado. Nos regocijamos mucho por ello... 
La vida y obra de Víctor Pimentel Gurmendi forman parte importante de la historia viva de la conservación del patrimonio cultural en el Perú. Como afirma Beingolea en la selección biográfica que narra los capítulos más trascedentes de la vida del viejo maestro, "A sus 87 años, VPG continúa vigente y activo en la defensa del patrimonio monumental, como parte del patrimonio cultural y natural, mantiene firme su postura ética, capaz de soslayar sus propios intereses personales. Defiende una visión integradora, inclusiva, ecuménica del patrimonio y del ser humano que constituye su origen y quien la da finalidad y sentido" (p. 16). El libro que reseñamos trae a las nuevas generaciones el pensamiento de VPG y, por ese hecho, constituye un aporte trascendental de cara a la recuperación del rol del patrimonio en el Perú, labor a la que VPG ha contribuido de manera notable.

Resulta pues que el libro tiene varios méritos. Uno de ellos es el homenajear a VPG, hecho que nos satisface doblemente pues se trata del presidente honorario de As. ICOMOS Perú, y uno de los firmantes de lo que podría considerarse el acto fundacional de la teoría moderna de la conservación, la Carta de Venecia.

VPG trajo las teorías de la conservación en las que se formó en Italia y las plasmó en hechos como la restauración de la Casa Garcilaso en el Cusco, luego de sostener un debate con el célebre Emilio Harth-Terré. Este hecho marca en el Perú el inicio de la conservación según principios acordes a las doctrinas internacionales. La obra fue reconocida con el Hexágono de Oro en la Primera Bienal de Arquitectura, como refiere Beingolea en su texto biográfico.

Mediante una entrevista, Beingolea nos presenta una "Breve autobiografía autoinducida" de VPG (primer capítulo del libro), en la que recuerda difíciles momentos de su infancia y juventud. Dedica especial atención al recuerdo de sus maestros en el antiguo Departamento de Arquitectura de la Escuela Nacional de Ingenieros: menciona a Jorge MueIle, Fernando Belaúnde y Carlos Morales. Especial es el recuerdo de Mario Bianco, con quien colaboró en la construcción de la sede del Departamento de Arquitectura de la Escuela de Ingenieros (DAENI).

De su etapa en Italia, Pimentel recuerda siempre a Carlos Ceschi, quien le permitiría participar en las obras de restauración del Coliseo y del Foro Romano. En

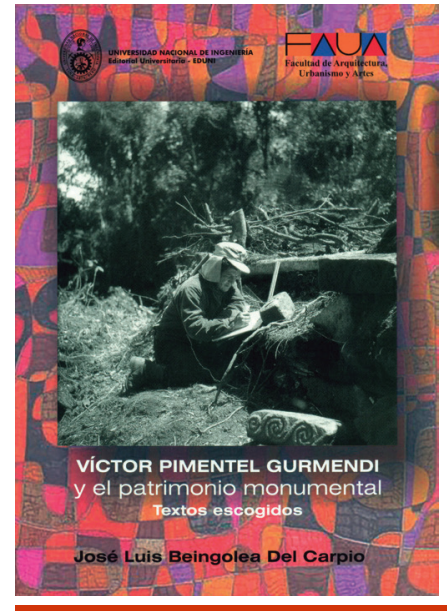

\section{VÍCTOR PIMENTEL GURMENDI Y EL PATRIMONIO MONUMENTAL. TEXTOS ESCOGIDOS}

JOSÉ LUIS BEINGOLEA DEL CARPIO

EDITORIAL UNIVERSITARIA DE LA UNIVERSIDAD NACIONAL DE INGENIERÍA, LIMA-PERÚ, 2015.

RESEÑA DE ALBERTO MARTORELL CARREÑO

otro texto de los seleccionados por Beingolea, "Crónicas desde el Coliseo", VPG explica parte de la relación anímica con el patrimonio que el paseo por la mágica Roma despierta: "Muchas veces me ocurre que después de haber recorrido todo aquél estupendo panorama, mi vista se detiene en algún pequeño particular contemplando el cual detenidamente, me sirve para serenar mi ánimo y dialogar con mis pensamientos de manera más luminosa" (p. 176).

Es difícil la tarea de recopilar el pensamiento de alguien que no solo tiene una vasta producción intelectual, sino que estuvo y fue actor de un momento fundacional en una corriente de pensamiento, en este caso la conservación del patrimonio cultural en el Perú. Beingolea asume ese reto luego de una ardua tarea de selección de textos y largas conversaciones con el maestro en su domicilio, sobre las que el propio VPG nos ha platicado varias veces.

El libro contiene seis secciones. La primera consiste en una visión biográfica de la que antes comentamos la autobiografía inducida, que se complementa mediante textos referentes a distintos momentos, elaborados por Paulo Ormindo de Acevedo, Fernando Belaúnde Terry y Silvio Mutal. En la segunda sección, "Memorias", es la pluma de VPG la que, en textos de la época, reseña a personajes como Héctor Velarde o el propio Arq. Fernando Belaúnde, y se refiere al Fondo Cultural Documental de la Arquitectura y Urbanismo (proyecto que VPG ha venido tratando de consolidar sin recibir suficiente apoyo institucional por parte del Colegio de Arquitectos), y la construcción del pabellón de la FAUA-UNI.
Tal vez la parte más significativa de la obra reseñada sea la sección tercera, "Conceptos y críticas", que contiene un total de 20 artículos en los que se puede percibir el pensamiento de VPG respecto al patrimonio cultural. Preocupa constatar que en fechas tan cercanas como 1956 el entonces joven arquitecto manifestara que "Estamos acostumbrados a leer y sentir muchos justificados lamentos por el estado de abandono en que se encuentra nuestro patrimonio artístico y cultural" (p. 51). La voz de VPG ha sido significativa para que grandes bienes de nuestro patrimonio hayan Ilegado hasta nuestros tiempos, pero evidentemente hay que seguir su noble ejemplo en la defensa de otros bienes olvidados y en proceso de desaparecer.

Las secciones $\mathrm{D}$ y $\mathrm{E}$, tituladas "Proyectos y propuestas" e "Historia de la Restauración" respectivamente, incluyen artículos fruto de la experiencia y conocimientos de VPG. El libro, de lectura obligatoria para los profesionales de la conservación, cierra con una sexta sección llamada "Crónicas", en la que se presentan las apreciaciones de Pimentel sobre lugares de Italia, como el Coliseo Romano, y de diversos puntos del Perú, como Conayca, Arequipa, Sajsahayhuamán, Casma, Río Abiseo, Zaña y Caral-Supe, donde VPG sigue siendo un asesor activo y de opinión altamente especializada. 


\section{ESTADO DEL ARTE DEL PATRIMONIO, LA PLANIFICACIÓN URBANA Y EL DESARROLLO SOSTENIBLE DE LA REGIÓN CUSCO}

\author{
FUNDACIÓN UNIVERSITARIA PARA \\ LA INVESTIGACIÓN, DESARROLLO, CIENCIA \\ Y CULTURA SAN ANTONIO ABAD DEL CUSCO \\ (FUNSAAC], CONVENIO CIUF/CUD - UNSAAC, \\ CUSCO-PERÚ, 2014.
}

RESEÑA DE JOSÉ HAYAKAWA CASAS

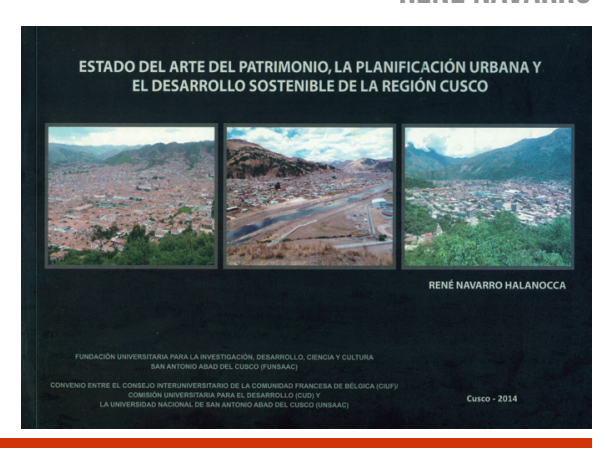

Esta publicación aparece en el marco del convenio sostenido por la Universidad Nacional san Antonio Abad del Cusco y el Conseil Interuniversitaire de la Communauté Française de Belgique, como expresión de la voluntad de "...impulsar la investigación regional y alcanzar respuestas que contribuyan al desarrollo científico, tecnológico, social, cultural y económico local" (p. 7), como lo menciona el mismo autor. Precisamente, este libro aborda la labor de realizar una examinación del avance y las perspectivas en la implementación de los preceptos del desarrollo sostenible en los ámbitos de la planificación urbana y de la conservación del patrimonio en la región del Cusco. Para ello, el autor toma como base la información entregada en la Actividad 05, referida al Estudio del Arte del Patrimonio, la Planificación Urbana y el Desarrollo Sostenible, actividad creada por el Convenio CIUF-UNSAAC entre 2010 y 2011.

Un aporte valioso de esta publicación radica en que ante un escenario de ausencia de bibliografía de rigor y calidad elaborada a partir de investigaciones serias referidas a las materias de estudio, se optó por (re)construir un estado del arte del patrimonio y la planificación urbana. Ello luego fue complementado por la realización de un estudio de tipo exploratorio, con abundante recolección de información y pesquisas de campo en una muestra de ciudades previamente seleccionadas de la Región del Cusco. En las referidas ciudades se aplicaron diversos indicadores que contribuyeron a medir los avances del desarrollo sostenible en la realidad urbana y en la realidad patrimonial, respectivamente. En esa línea y en coherencia con lo explicado anteriormente, este libro consta de cuatro partes que lo estructuran: 1) Problema de estudio y método, 2) Carac- terización de la región del Cusco, 3) Acopio de información y análisis de indicadores, y 4) Hallazgos y recomendaciones.

En la primera parte, el autor abunda en detalles acerca del proceso de formulación del problema y la explicación del método de estudio. Asimismo, argumenta las razones por las cuales se decide tomar como ciudades-muestra al Cusco, Sicuani, Quillabamba, Uurubamba, Calca y Pisaq. También define las respectivas y precisas variables e indicadores a utilizar en el diagnóstico, que luego serían utilizadas en la búsqueda de información bibliográfica y de campo.

En la segunda parte, el Arq. Navarro desarrolla con mayores detalles la caracterización espacio-territorial (geomorfología, ecología, clima, cuencas hidrográficas, vulnerabilidad ambiental), socioeconómica (corredores y flujos económicos, redes urbano regionales, sistema vial terrestre, transporte aérea, red de ferrocarril), y urbana y patrimonial (demografía, migración, sistema urbano, patrimonio cultural prehispánico, patrimonio cultural virreinal) de la Región del Cusco.

En la tercera parte, presenta la información recabada en las ciudades-muestra, tal es el caso de la ciudad mayor del Cusco, las ciudades intermedias de Quillabamba y Sicuani, las ciudades menores de Urubamba, Calca y Pisaq. Para el análisis, utiliza datos de entrada como la ubicación y el territorio, y agrupando indicadores por afinidad temática, tales como los "indicadores generales": predisposición institucional, política y económica, disponibilidad del aparato administrativo; oficina, recurso humano calificado, técnico y logístico; disponibilidad de un plan urbano y de conservación del patrimonio; gestión política, institucional, urbana y ambiental.
Asimismo, considera los "indicadores de diagnóstico de la planificación urbana" : territorialidad urbana o rural; lineamientos de ordenamiento territorial y regulación del crecimiento urbano; protección del paisaje natural y cultural; previsión urbana de ocupación de zonas no aptas, control, y mitigación de riesgos; generación de contaminantes, residuos y su tratamiento; uso racional del suelo urbano y correspondencia con las actividades urbanas, conectividad vial y transporte, reserva de áreas para equipamiento urbano; formulación de programas de vivienda y previsión de infraestructura básica; programas a nivel de renovación, rehabilitación urbana y tratamiento de espacios públicos.

También toma en cuenta los "indicadores de diagnóstico de la conservación del patrimonio urbano-arquitectónico": impacto de la urbanización en el paisaje natural y cultural; impacto de la urbanización en el patrimonio arqueológico; tratamiento de los problemas urbanísticos en los tejidos urbanos con valor histórico; impacto de la arquitectura contemporánea en los tejidos urbanos con valor histórico; impacto del turismo en los tejidos urbanos con valor histórico.

Además, destacan los "indicadores de diagnóstico del desarrollo sostenible": políticas de protección del territorio, recursos naturales, suelos y el paisaje; capacidad regenerativa del ecosistema, recursos naturales y energético; ambientes construidos y públicos sostenibles; pluralidad de usos y actividades urbanas, eficiencia de la conectividad vial y transporte; participación de las instituciones y la población en el desarrollo de la ciudad y la protección del patrimonio cultural; cohesión social.

Finalmente, se presentan los principales hallazgos y recomendaciones, los cuales se plantean siguiendo la misma estructura temática (planificación urbana, conservación del patrimonio, desarrollo sostenible). Aquí es donde se echa de menos un mayor esfuerzo de síntesis y articulación de los diferentes datos organizados monotemáticamente, que permitirían una lectura multidimensional de una realidad compleja, dinámica e interdependiente.

Dicho esto, solo cabe invitarlos a sumergirse en un buen estudio que (re)construye una mirada panorámica y respaldada de la relación entre la planificación urbana, la conservación del patrimonio y el desarrollo sostenible, componentes estratégicos y de valor contemporáneo para la(s) realidad(es) de la Región del Cusco. 
Revista chilena editada por el Centro Nacional de Conservación y Restauración (CNCR) - con el auspicio de la Dirección de Bibliotecas, Archivos y Museos (DIBAM), y el Ministerio de Educación- dedicada a la investigación, la conservación y la gestión del patrimonio. Anualmente aparece en edición impresa, y accesible desde el enlace: http://www.cncr.cl/611/ w3-articlr-62767.html

Entre 1988 y 2010 estuvo a cargo de la Arq. Magdalena Krebs, directora entonces del CNCR, quien hoy cuenta, en su Comité editorial, junto con el Arq. José de Nordenflycht del Consejo de Monumentos Nacionales y la Dra. Mónica Bahamondez, su actual directora. Es editora general la Mg. Roxana Seguel, conservadora de Arqueología del CNCR, quien en Lima participó al reciente Convenio sobre Conservación de Momias, y en esta publicación es coautora de un artículo relativo a su especialidad en la "Selección de notas breves".

Los restantes abordan problemáticas referentes al patrimonio y la conservación, mediante estudios de casos, informes técnicos y análisis específicos. Algunos diferentes al ámbito de Devenir -el patrimonio edificado- $y$, sin embargo, interesantes: i) "Evaluación de la plastificación como técnica para consolidar material arqueológico"; iii) "Modelos de restauración y conservación de material fílmico en Chile"; iv) "Un pintor recupera a su modelo. Identificación de retrato"; v) "Efectos de la tracción mecánica sobre el soporte textil de una antigua obra pictórica". Además, otros informes puntuales: vi) "Intervención de 36 dibujos naturalistas del Museo de Historia Natural de Valparaíso"; vii) "Manuscrito italiano"letra capital 'd' decorada'"' y viii) "Estudio de materiales de la escultura Buda Dainichi Nyorai".

Presentan mucho interés y mayor proximidad temática (ii) "Las Azudas: condición actual de una singular expresión hidráulica que identifica la localidad de Larmahue, en pleno valle central chileno", de los arquitectos Antonio Sahady, Marcelo Bravo y Carolina Quilodrán, cuyo proyecto fue financiado por el Fondo $\mathrm{Na}$ cional de Desarrollo Científico y Tecnológico. Las 'Azudas' son ruedas con aspas de madera o norias que elevan el agua movidas por la corriente en la localidad vinícola de la comuna chilena de Pichidegua, un pintoresco sistema que otorga valor patrimonial al paisaje. Se empezaron a emplear a inicios del siglo pasado en Chile; hoy están en desuso y en peligro de desaparecer. Fueron identificadas 41 azu-

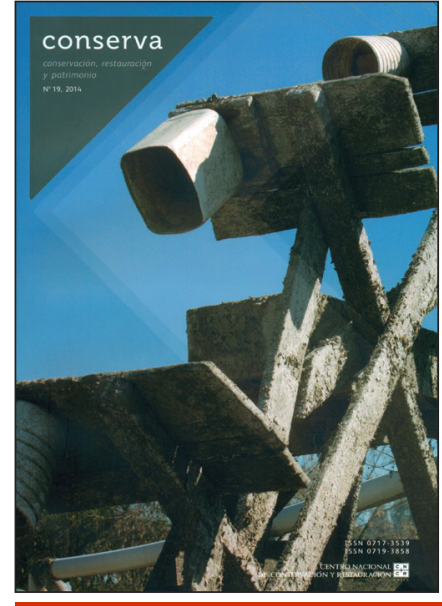

CONSERVA. REVISTA DE CONSERVACIÓN, RESTAURACIÓN Y PATRIMONIO N 19

\author{
CENTRO NACIONAL DE CONSERVACIÓN \\ Y RESTAURACIÓN \\ CENTRO NACIONAL DE CONSERVACIÓN Y RESTAURACIÓN \\ [CNCR], SANTIAGO DE CHILE, 2014.
}

RESEÑA DE LEONARDO MATTOS-CÁRDENAS

das, clasificadas según su estado de conservación. Se les desea valorizar mediante un programa de gestión de recursos para rutas turístico-culturales inspiradas en la experiencia de la región española de Murcia, donde como en otras zonas del Mediterráneo -incluyendo Italia, no solo insular- se adoptó esta técnica árabe.

Igualmente, el x) “Diagnóstico del paisaje cultural de la Cuenca del río lbáñez, región de Aysén, Chile" de Bernardita Ladrón de Guevara, Darío Toro, Rafael Prieto y Carolina Chávez, del CNCR, realizado para un programa de puesta en valor del patrimonio del BID. Un paisaje patagónico de particular continuidad e indisociabilidad con ocupación prehispánica y pinturas rupestres, de colonización reciente y vocación ganadera, además del volcán Hudson y el lago General Carrera, que dan 'identidad territorial' a esa región (247,000 ha.), actualmente en riesgo por la carencia de herramientas que identifiquen y valoren los componentes patrimoniales del paisaje. Destacan los referentes del Convenio Europeo del Paisaje (Consejo de Europa 2000) y el "Catálogo del paisaje de Cataluña” (Nogué y Sala 2006) ${ }^{1}$, los cuales valoran el territorio con un enfoque multifactorial, y consideran su dimensión social, cultural y económica.

Son paisajes de la memoria, la identidad, la vida social, espiritual, la dimensión simbólica y la vida productiva que es necesario identificar -mediante talleres participativos realizados junto con sus habitantes- para poder construir un plan adecuado para dicha cuenca. Para ello,

\footnotetext{
1. Disponible en: http://dialnet.unirioja.es/ descarga/articulo/3027474.pdf
}

esta ha sido subdividida en tres 'unidades de paisaje':

\begin{abstract}
- 'Paisaje antrópico': con mayor población, infraestructuras urbanas y actividades productivas.
\end{abstract}

- 'Paisaje relictual': personificación del gaucho patagónico, ganadería, agricultura y uso turístico de praderas, bosques, lagos, y el cerro Castilloc como hito de identidad. Sus directrices preservan su rol amortiguador y conector en el sistema de cuencas.

- 'Paisaje elemental': inaccesible y deshabitado, con zonas de bosque y el volcán Hudson, cuya cumbre helada domina la unidad, valorada por su pristinidad. Requiere una gestión ambiental y productiva sustentable del paisaje, que rompa su aislamiento y preserve los ecosistemas.

Aunque - afirman los autores- mientras no se llegue a la evaluación final, será imposible determinar cuál será su meta final; sin embargo, ya ofrece una mirada sistémica del territorio abordado desde su paisaje.

La revista además, incluye referencias a otros ensayos, "Antecedentes y consideraciones para la conservación del patrimonio cultural en el siglo XXI" (Quirós \& Casas, 2011) ; “El patrimonio era otra cosa" (Criado-Boado \& Barreiro, 2013) y "Concepto y gestión del patrimonio local" (Prats, 2005), donde el todo es concebido como un 'constructo social".

Esta revista reúne contribuciones sobre la complejidad del patrimonio, desde ciencias sociales humanas y básicas que tienden a una gestión participativa, integral y sustentable, aunque se eche de menos una mayor profundidad histórica en algunas contribuciones. 